\title{
Protein hydrolysates and recovery of muscle damage following eccentric exercise
}

\author{
${ }^{1}$ Dale M.J., ${ }^{1}$ Thomson R.L., ${ }^{1}$ Coates A.M., ${ }^{1,2}$ Howe P.R.C., ${ }^{3}$ Brown A., \\ ${ }^{1 *}$ Buckley J.D.
}

${ }^{1}$ Alliance for Research in Exercise, Nutrition and Activity (ARENA), Sansom Institute for Health Research and School of Health Sciences, University of South Australia, Adelaide, Australia; ${ }^{2}$ Clinical Nutrition Research Centre, University of Newcastle, Newcastle, Australia; ${ }^{3}$ Murray Goulburn Co-operative Co. Ltd, Melbourne, Australia

*Corresponding author: Professor Jonathan D Buckley, Alliance for Research in Exercise, Nutrition and Activity (ARENA), Sansom Institute for Health Research, University of South Australia, GPO Box 2471, Adelaide 5001, Australia

Submission date: July 18, 2014; Acceptance date: January 22, 2015; Publication date: January 31,2015

\section{ABSTRACT}

Background: A whey protein hydrolysate (NatraBoost $\mathrm{XR} ; \mathrm{WPH}_{\mathrm{NB}}$ ) has been shown to speed repair muscle damage. We sought to determine whether this benefit is specific to this hydrolysate to evaluate a marker for quality control.

Methods: Three hydrolysates of the same whey protein isolate (WPI) were prepared $\left(\mathrm{WPH}_{\mathrm{NB}}, \mathrm{WPH}_{1}\right.$ and $\left.\mathrm{WPH}_{2}\right)$. Isometric knee extensor strength was measured in 39 sedentary male participants before and after 100 maximal eccentric contractions of the knee extensors to induce muscle damage. Participants were then randomised to consume $250 \mathrm{ml}$ of flavoured water ( $\mathrm{FW}, \mathrm{n}=9)$, or $250 \mathrm{ml}$ of $\mathrm{FW}$ containing $25 \mathrm{~g}$ of either NatraBoost XR (n=3), $\mathrm{WPH}_{1}$ $(n=9), \mathrm{WPH}_{2}(\mathrm{n}=9)$ or WPI $(\mathrm{n}=9)$. Strength was reassessed over the next seven days while the supplements were consumed daily. Fibroblasts were cultured for $48 \mathrm{hr}$ in the presence of the different hydrolysates, WPI, saline or fetal bovine serum to ascertain effects on cell proliferation.

Results: Strength was reduced in all treatment groups after eccentric exercise $(\mathrm{P}<0.001)$. Strength recovered steadily over 7 days in the FW, WPI, $\mathrm{WPH}_{1}$ and $\mathrm{WPH}_{2}$ treatment groups $(\mathrm{P}<0.001)$, with no difference between treatments $(\mathrm{P}=0.87)$. $\mathrm{WPH}_{\mathrm{NB}}$ promoted faster strength recovery compared with the other treatments $(\mathrm{P}<0.001)$. Fibroblast proliferation was greater with $\mathrm{WPH}_{\mathrm{NB}}$ compared with saline, WPI or the other hydrolysates $(\mathrm{P}<0.001)$.

Conclusions: Promoting recovery from muscle damage seems unique to $\mathrm{WPH}_{\mathrm{NB}}$. In vitro fibroblast proliferation may be a useful marker for quality control. It is not clear whether effects on fibroblast proliferation contribute to the in vivo effect of $\mathrm{WPH}_{\mathrm{NB}}$ on muscle damage.

Keywords: tissue repair, fibroblast proliferation, strength recovery, whey protein 


\section{BACKGROUND:}

Contraction of skeletal muscle, particularly eccentric contraction during as the skeletal muscle is lengthening, causes muscle damage. This damage is followed by an acute inflammatory response: swelling, pain, and a reduction in muscle strength that can persist for up to one week [1]. The pain and swelling as well as the reduction in muscle strength may impede subsequent exercise training or exercise performance; thus methods for accelerating recovery between training sessions have received much attention in the athletic training literature and numerous post-exercise recovery modalities [2].

Proteins are comprised of long chains of amino acids (primary structure) that are linked by peptide bonds and form secondary structures (e.g. $\alpha$-helix, $\beta$-pleated sheet) which then fold to form tertiary structures or become linked with other peptide chains to form quaternary structures [3]. Protein hydrolysates can be produced by chemically unfolding proteins and enzymatically hydrolysing the peptide bonds at various points in the primary structure to produce peptides containing varying numbers and combinations of amino acids. The treatment of proteins in this way essentially results in "pre-digestion", which has been proposed to facilitate subsequent amino acid absorption when consumed, resulting in a more rapid increase in circulating amino acid concentrations with a subsequent rapid increase in protein synthesis [4]. Alternatively, it has been proposed that hydrolysis of proteins may generate peptides that have bioactive properties, some of which might provide various benefits for human health [4].

Previously, hydrolysates of various proteins have been used to promote tissue repair after surgery [5], to speed recovery of tissue following burns [6] and to assist with repair of various other forms of trauma to a range of soft tissues [4]. In addition, we recently demonstrated that hydrolysis of a whey protein isolate (WPI) resulted in the formation of a whey protein hydrolysate (WPH) that was effective in speeding repair of skeletal muscle damage induced by eccentric exercise, resulting in a more rapid recovery of muscle strength (NatraBoost XR, $\mathrm{WPH}_{\mathrm{NB}}$, Murray Goulburn Co-operative Co. Ltd, Melbourne, Australia) [7]. $\mathrm{WPH}_{\mathrm{NB}}$ was selected for evaluation in our initial study because it was shown to elicit the greatest proliferative response when incubated with fibroblasts in vitro (unpublished data), and because fibroblasts are responsible for laying down new tissue matrix during tissue repair their proliferation is an important step in the tissue repair process, including in skeletal muscle [8]. However, it is not clear whether these effects on in vitro fibroblast proliferation and in vivo repair of skeletal muscle damage are unique to this particular hydrolysate, or whether other protein hydrolysates also elicit similar effects.

While fibroblast proliferation is an important step in tissue repair, in vitro effects on fibroblast proliferation may not necessarily be directly related to in vivo effects on tissue repair. Nevertheless, if protein hydrolysates that promote tissue repair in vivo also promote fibroblast proliferation in vitro, regardless of whether or not the two processes are directly mechanistically linked, monitoring of in vitro fibroblast proliferation might provide a useful index of the potential for in vivo efficacy of a hydrolysate in terms of its potential for promoting tissue repair. The hydrolysates produced during enzymatic hydrolysis are dependent on the conditions under which the hydrolysis proceeds, with the $\mathrm{pH}$ at which the hydrolysis is performed influencing the activity of the enzymes employed and thus the number of peptide bonds that are hydrolysed and the number of peptides that are produced. The duration of hydrolysis also influences the extent of hydrolysis of the proteins, and the nature of the hydrolytic enzymes employed not only influences which peptide bonds are 
cleaved (and thus what peptides are formed) but also the speed with which the hydrolysis proceeds and thus the extent of hydrolysis that occurs. Varying any of these chemical parameters during the hydrolytic process can result in the formation of products that are hydrolysed to either a greater or lesser extent and contain peptides of differing configurations with potential implications for differential biological effects [4]. Therefore, variations in the process of manufacturing $\mathrm{WPH}_{\mathrm{NB}}$ may influence its efficacy for promoting repair of skeletal muscle tissue and monitoring of effects on in vitro fibroblast proliferation might provide a useful marker of quality control.

The aim of this study was to determine a) whether the ability to speed repair the muscle damage induced by eccentric exercise is a unique property of $\mathrm{WPH}_{\mathrm{NB}}$ or whether this is common to other whey protein hydrolysates and, b) whether in vitro effects on fibroblast proliferation are associated with the efficacy of muscle repair in vivo, and might therefore be useful as a marker of quality control that could be monitored during the manufacture of $\mathrm{WPH}_{\mathrm{NB}}$.

\section{METHODS}

Experimental Overview. $\mathrm{WPH}_{\mathrm{NB}}$ was manufactured from WPI using a proprietary hydrolytic method [9]. Two other hydrolysates were manufactured using slight variations of this method that involved using longer durations of hydrolysis, altering the method of drying used to convert from a liquid for to a powder, and for one of the hydrolysates starting with WPI with a slightly different protein composition.

The in vitro activity of the hydrolysates was tested by culturing fibroblasts in the presence of the hydrolysates and measuring effects on fibroblast proliferation. Fibroblasts were also cultured in the presence of normal saline (negative control), unhydrolysed WPI (negative control) and fetal bovine serum (FBS) (positive control).

To test in vivo activity of the hydrolysates, sedentary males (22 yr, $176 \mathrm{~cm}, 79 \mathrm{~kg}$ ) had their isometric knee extensor strength assessed prior to completing 100 maximal eccentric contractions of their knee extensor muscles to induce muscle damage. Isometric knee extensor strength was then reassessed and participants were randomised in a double-blind manner (via minimisation [10] based on their baseline knee extensor strength) to consume $250 \mathrm{ml}$ of flavoured water (FW, n=9) or $250 \mathrm{ml}$ of $\mathrm{FW}$ containing $25 \mathrm{~g}$ of $\mathrm{WPH}_{\mathrm{NB}}(\mathrm{n}=3)$, the two other whey protein hydrolysates $\left(\mathrm{WPH}_{1} ; \mathrm{n}=9\right), \mathrm{WPH}_{2}(\mathrm{n}=9)$ or unhydrolysed WPI $(\mathrm{n}=9)$. Strength was then reassessed at 1, 2, 6, 24, 48, 72 and 168 hours (i.e. 7 days) post-exercise while participants consumed the appropriate supplement once daily. Recovery of isometric torque was compared between treatments as a marker of repair of muscle damage [1].

In vitro assessment: BalbC $3 \mathrm{~T} 3$ fibroblasts were seeded, in duplicate, into 96-well plates in cell-culture medium containing $10 \%$ fetal bovine serum (FBS) and maintained in culture overnight at $37^{\circ} \mathrm{C}$. Following the overnight culture, the serum-containing medium was removed from all but two wells (FBS was retained in these wells as a positive control) and cells were stimulated in serum-free medium with either vehicle control $(0.9 \%$ saline $)$ or 2 $\mathrm{mg} \cdot \mathrm{ml}^{-1}$ of WPI, or one of the three hydrolysates being evaluated. Cells were maintained in culture for an additional 48 hours after which cell proliferation was quantified by Alamar Blue staining. 
In vivo assessment: Thirty-nine sedentary males (18-30 years) were recruited via public advertisement. Participants were excluded from the study if they reported current or recent (prior 3 months) participation in physical activity more than once a week for the purpose of improving or maintaining their fitness, if they had undertaken any form of resistance training of the lower limbs in the prior 3 months, had any form of knee, quadriceps or other musculoskeletal or medical problem that would prevent performance of the exercise required for the study, if they had experienced significant soreness in the quadriceps muscles in the 3 months prior to the study, or if they had exhibited any prior allergic response or sensitivity to dairy foods, or were intolerant of dairy proteins or lactose.

Participants were required to abstain from alcohol from 48 hours prior to commencement of the study until study completion, and were directed not to use any form of analgesic or anti-inflammatory medication during the study. Participants were habituated to the experimental procedures the week prior to participation and presented to the laboratory the morning after an overnight fast. The study and procedures were conducted in accordance with the Declaration of Helsinki and approved by the Human Research Ethics Committee of the University of South Australia. All participants provided written informed consent prior to participation.

Knee extensor torque of the right leg was determined using an isokinetic dynamometer (Biodex System 4, Biodex Medical Systems Inc, NY). Participants performed one set of three submaximal isometric contractions as a warm-up with a one-minute rest between repetitions. After a further two-minute rest three maximal isometric contractions was performed with a one-minute rest between repetitions. The maximum torque achieved was taken to be the measured value for knee extensor strength. Test-retest reliability for assessment of maximal knee extensor torque in this way in our laboratory is excellent, with an intraclass correlation coefficient $\pm 95 \%$ confidence interval of $0.92 \pm 0.05$, based on two testing sessions separated by $1-2$ weeks on 46 volunteers.

To induce muscle damage, 100 maximal voluntary eccentric isokinetic contractions of the knee extensors of the right leg were performed. During the active (i.e. eccentric) phase of the contractions, participants resisted movement of the lever arm of the dynamometer with maximum effort. The arm of the dynamometer moved through a $90^{\circ}$ range of motion at an angular velocity of $45^{\circ} . \mathrm{s}^{-1}$. During the passive phase of the motion, the dynamometer returned the participant's leg to the starting position at a speed of $10^{\circ} . \mathrm{s}^{-1}$. During this return phase the participants were instructed to relax and allow this repositioning to occur without resistance.

$\mathrm{WPH}_{\mathrm{NB}}$ was prepared by a patented method and freeze dried [9]. The other two hydrolysates $\left(\mathrm{WPH}_{1}\right.$ and $\left.\mathrm{WPH}_{2}\right)$ were prepared using the same hydrolytic enzymes, but both had longer hydrolysis times, were spray dried rather than freeze dried, and the raw material used to prepare $\mathrm{WPH}_{2}$ contained a lower level of $\alpha$-lactalbumin. The level of hydrolysis of all hydrolysates was $1.1-1.3 \%$.

The supplements were provided to participants in pre-weighed sachets in powder form. The sachets each weighed $30 \mathrm{~g}$ and contained $25 \mathrm{~g}$ of the supplement powder, $1.5 \mathrm{~g}$ skim milk powder (MG Nutritionals, Parkville, Australia) and $3.5 \mathrm{~g}$ of a sucrose-containing flavour (IFF Flavouring, Melbourne, Australia). For the flavoured water control $30 \mathrm{~g}$ of the sucrosecontaining flavor was provided and added to the water. Supplements were added to $250 \mathrm{ml}$ of water and mixed thoroughly prior to their consumption. The supplement sachets were labeled alphabetically and the investigators and participants were blinded to the treatments being 
delivered. The supplements containing protein were isocaloric and contained the same amount of total protein.

Statistics. The normality of data was assessed using the Shapiro-Wilk test prior to applying parametric analysis. In vitro data were assessed using one-way analysis of variance (ANOVA, Statistical v5.1, StatSoft Inc., Tulsa, Okla., USA). For the in vivo data, two-way ANOVA with repeated measures was used to determine the effects of the supplements on the dependent measures over time. Where ANOVA demonstrated a significant main effect posthoc analysis was conducted to identify differences between means using Tukey's test for Honestly Significant Differences. Statistical significance was set at a $\alpha$-level of $\mathrm{P}<0.05$. All data are shown as mean \pm SEM.

\section{RESULTS:}

In vitro assessment: With all treatments the proliferation of BalbC3T3 fibroblasts was significantly greater compared with the negative saline control $(\mathrm{P}<0.001)$ and significantly less compared with the $10 \%$ FBS positive control ( $\mathrm{P}<0.001$; Figure 1). Cell proliferation was approximately two-folds greater with $\mathrm{WPH}_{\mathrm{NB}}$ compared with the other protein hydrolysates and WPI $(\mathrm{P}<0.001) . \mathrm{WPH}_{1}$ was significantly greater than WPI $(\mathrm{P}=0.03)$, but not different from $\mathrm{WPH}_{2}(\mathrm{P}=0.08)$.

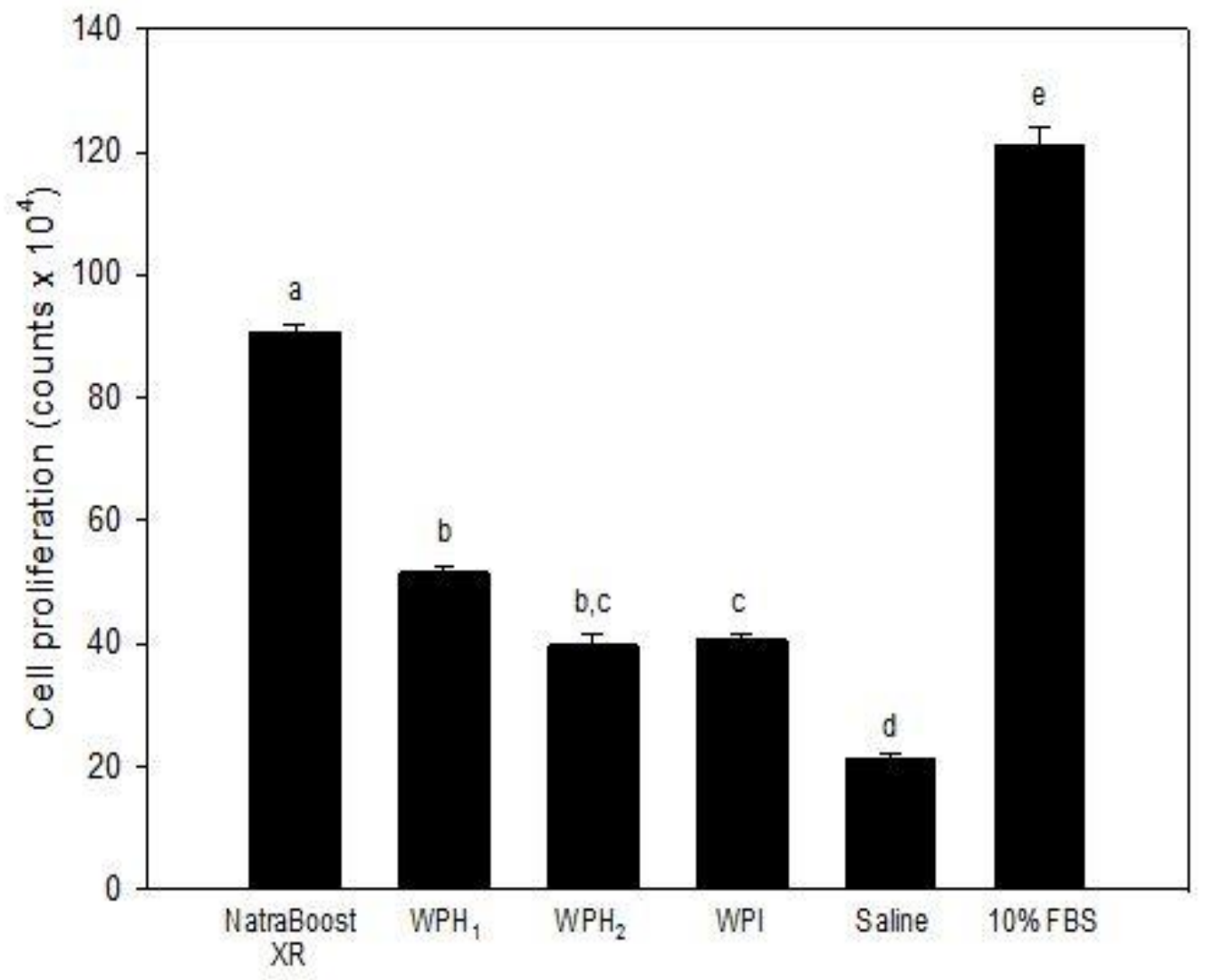

Figure 1. Cell proliferation of BalbC3T3 fibroblasts. Fibroblasts were cultured for $48 \mathrm{hr}$. at $37^{\circ} \mathrm{C}$ in the presence of $0.9 \%$ saline (negative control), or $2 \mathrm{mg} / \mathrm{ml}$ of either NatraBoost XR, two other hydrolysates $\left(\mathrm{WPH}_{1}\right.$ and $\left.\mathrm{WPH}_{2}\right)$, unhydrolysed whey protein isolate (WPI), or $10 \%$ fetal bovine serum (10\% FBS, positive control). Values with different superscript letters differ significantly $(\mathrm{P}<0.05)$. 
In vivo assessment: There was no difference in the maximum isometric torque (i.e. muscle strength) between groups at baseline (WPI $251.3 \pm 24.7 \mathrm{n} \cdot \mathrm{m}, \mathrm{WPH}_{1} 248.5 \pm 22.9 \mathrm{n} \cdot \mathrm{m}, \mathrm{WPH}_{2}$ $\left.270.0 \pm 16.0 \mathrm{n} \cdot \mathrm{m}, \mathrm{FW} 252.8 \pm 27.9 \mathrm{n} \cdot \mathrm{m}, \mathrm{WPH}_{\mathrm{NB}} 261.6 \pm 57.6 \mathrm{n} \cdot \mathrm{m} ; \mathrm{P}=0.97\right)$. Peak torque was decreased significantly in all treatment groups following the performance of 100 maximal eccentric muscle contractions ( $\mathrm{P}<0.001$ for time, Figure 2 ) with no difference in the magnitude of this reduction between groups $(\mathrm{P}=0.94$ for treatment $\mathrm{x}$ time). Torque production recovered steadily over the 7-day study period $(\mathrm{P}<0.001$ for time), with no difference in the rate of recovery between treatments $(P=0.13$ for treatment $x$ time), although there was a tendency for recovery to be more rapid with $\mathrm{WPH}_{\mathrm{NB}}$.

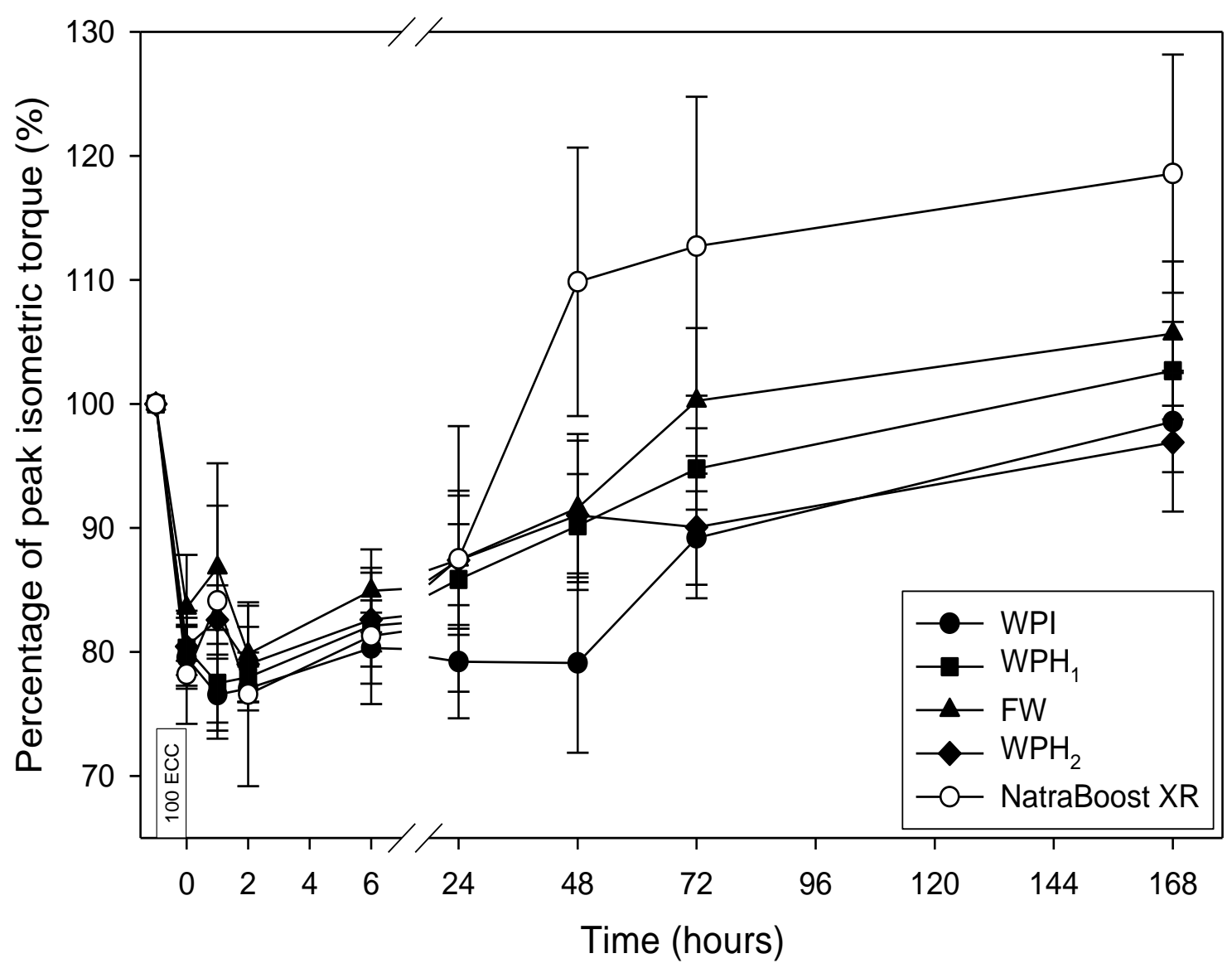

Figure 2. Peak isometric torque changes following eccentric exercise and supplementation for each condition. Percentage of peak isometric torque prior to and following the performance of 100 maximal eccentric contractions of the knee extensor muscles of the right leg and then supplementation with various supplements over a 7-day (i.e. $168 \mathrm{hr}$.) period. 100ECC - 100 maximal eccentric contractions of the knee extensors. WPI - unhydrolysed whey protein isolate, NatraBoost XR - Whey protein hydrolysate previously shown to speed recovery of peak isometric torque following eccentric exercise [7], two other hydrolysates $\left(\mathrm{WPH}_{1}\right.$ and $\left.\mathrm{WPH}_{2}\right), \mathrm{FW}$ - flavoured water.

Analysis of the control treatments (WPI, $\mathrm{WPH}_{1}, \mathrm{WPH}_{2}$ and $\mathrm{FW}$ ) indicated no differences in the rate of recovery of muscle strength $(\mathrm{P}=0.87$ for treatment $\mathrm{x}$ time $)$, so these treatments were pooled for comparison with $\mathrm{WPH}_{\mathrm{NB}}$. This analysis revealed a significant treatment $\mathrm{x}$ time interaction $(\mathrm{P}<0.001)$, indicating that the recovery of muscle strength was faster in 
participants who had been supplemented with $\mathrm{WPH}_{\mathrm{NB}}$ compared with the other treatments (Figure 3).

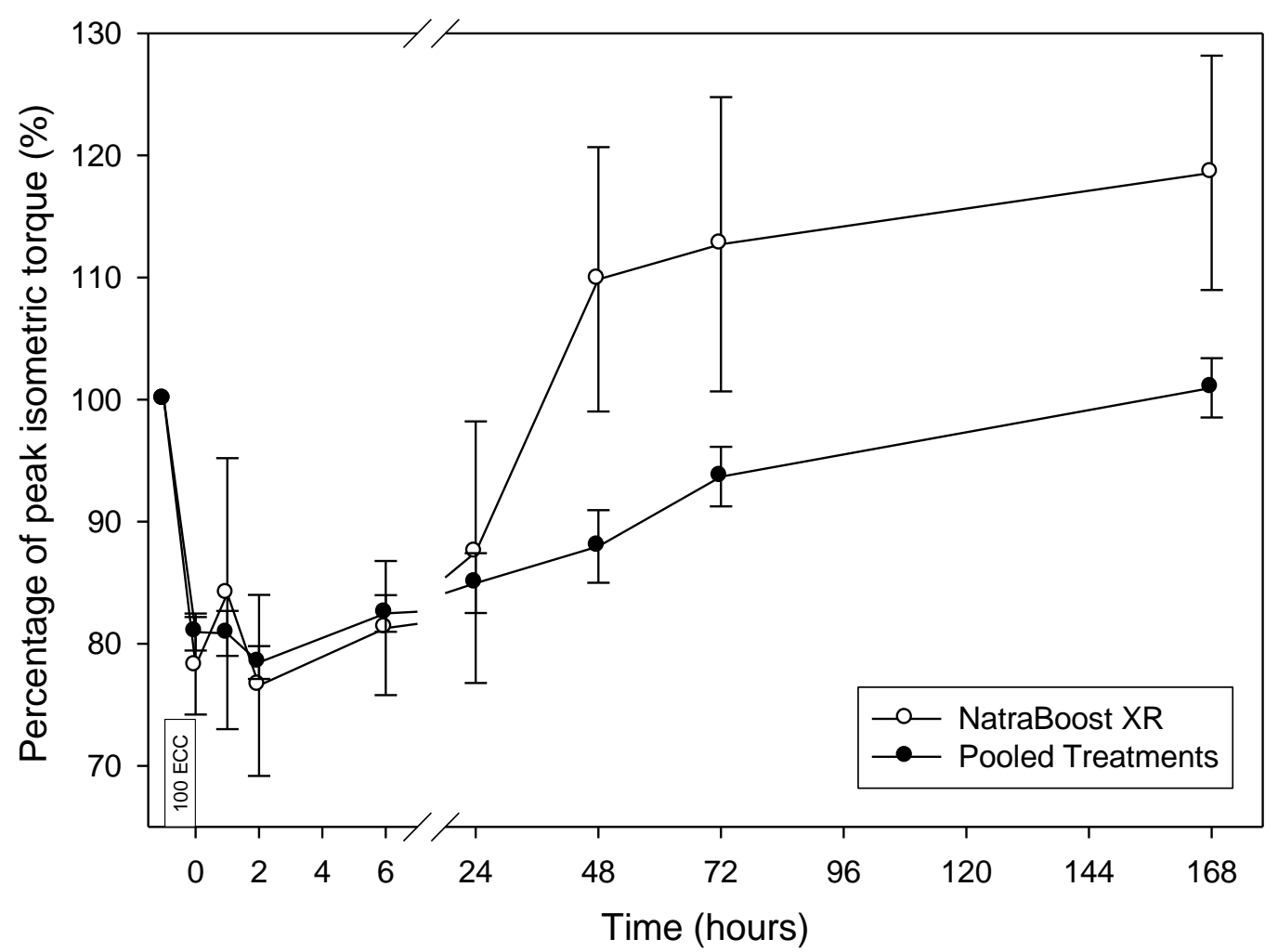

Figure 3. Peak isometric torque changes following eccentric exercise and supplementation for NatraBoost XR hydrolysate compared to pooled control treatments. Percentage of peak isometric torque prior to and following the performance of 100 maximal eccentric contractions of the knee extensors of the right leg and then supplementation with various supplements over a 7-day (i.e. $168 \mathrm{hr}$ ) period. Pooled treatments consisted of combined data for effects of whey protein isolate, two whey protein hydrolysates $\left(\mathrm{WPH}_{1}\right.$ and $\left.\mathrm{WPH}_{2}\right)$, and flavoured water. 100ECC - 100 maximal eccentric contractions of the knee extensors. NatraBoost XR - Whey protein hydrolysate previously shown to speed recovery of peak isometric torque following eccentric exercise [7].

\section{DISCUSSION:}

The principal finding of the current study was that different methods of preparing hydrolysates from the same, or similar, starting material resulted in markedly different in vitro and in vivo effects on fibroblast proliferation and repair of skeletal muscle damage, respectively. Indeed, the different methods of preparing the hydrolysates in the present study resulted in a loss of efficacy for promoting recovery of muscle torque. The implication of this is that care must be exercised when preparing a particular hydrolysate as even small changes in the processing method might result in the production of a different end product that does not elicit the desired outcome.

In order to demonstrate greater efficacy of the $\mathrm{WPH}_{\mathrm{NB}}$ in terms of speeding recovery of muscle strength it was necessary to pool the data from the other treatments to achieve statistical power. Indeed, pooling of data is the basis of the many meta-analyses which are commonly reported in literature [11], but such pooling to achieve statistical power is only appropriate provided certain criteria are met $[11,12]$. Pooling of data is only appropriate 
when principal treatment, diagnosis, clinical severity, and outcome events are similar. In the present study participants whose data were pooled were subjected to similar treatments (i.e. induction of muscle damage and then consumption of supplements with unknown efficacy for improving recovery of muscle strength, as opposed to the group treated with $\mathrm{WPH}_{\mathrm{NB}}$ which has previously been established to promote recovery of muscle strength in an adequately powered trial). The participants also had similar diagnosis, i.e. muscle damage induced by eccentric exercise; the clinical severity was similar, as the reduction in muscle torque did not differ between groups. Outcome events were also the same, i.e. recovery of muscle torque that did not differ between treatment groups. Thus, in this case it was appropriate to pool the data to increase statistical power and in doing so it appears that this provided a better estimate of efficacy given we have previously shown that the $\mathrm{WPH}_{\mathrm{NB}}$ supplement is efficacious for improving recovery of muscle damage. Indeed, random sampling errors of differing directions and magnitudes that might have occurred as a result of the small number of participants in each treatment group would have tended to cancel out when the groups were pooled, thus reducing the likelihood of type 1 error and providing a more accurate overall estimate of the treatment effect (Naylor, 1988). Nevertheless, while pooling the data provided confirmation that the $\mathrm{WPH}_{\mathrm{NB}}$ treatment is efficacious for speeding the recovery of muscle damage. The primary finding of the present study was that if the same/or similar starting material is hydrolysed then depending on the conditions under which the hydrolysis is performed the functionality of the hydrolysate can be substantially altered.

All of the hydrolysates in the present study were derived from similar base material, apart from a lesser amount of $\alpha$-lactalbumin in $\mathrm{WPH}_{2}$, but longer durations of hydrolysis were employed compared with $\mathrm{WPH}_{\mathrm{NB}}$ and were spray dried rather than freeze dried, resulting in the production of distinct hydrolysates. $\mathrm{WPH}_{\mathrm{NB}}$ elicited a much greater in vitro proliferation of fibroblasts than the other hydrolysates and the unhydrolysed WPI and also facilitated a more rapid recovery of muscle strength compared with the other supplements. However, while an association between greater in vitro fibroblast proliferation and more rapid recovery of muscle strength was evident, this cannot be interpreted as causative as identification of the specific bioactive(s) has not been achieved, nor is there evidence that the bioactive(s) survive the digestive process and are delivered to the target tissue in a dosedependent manner. However, findings such as in the present study do inform directions for future research to further evaluate potential mechanistic relationships between the in vitro and in vivo findings.

While the mechanism by which $\mathrm{WPH}_{\mathrm{NB}}$ accelerated recovery from muscle damage remains to be elucidated, it has been proposed [13] that protein hydrolysates increase delivery of amino acids to the muscle as a result of a more rapid bioavailability due to the predigestion of the protein, with the amino acids increasing muscle protein synthesis and reducing protein degradation. Plasma amino acid concentrations were not measured in the present study, but Farnfield et al [14] demonstrated an attenuation of the increase in plasma amino acid concentrations with consumption of hydrolysed whey protein compared with unhydrolysed whey protein, suggesting that not all whey protein hydrolysates increase bioavailability of amino acids. Indeed, the fact that the different whey protein hydrolysates assessed in the present study were all hydrolysed to a similar extent $(1.1-1.3 \%)$ also argues against the effect of the $\mathrm{WPH}_{\mathrm{NB}}$ being mediated by a difference in amino acid bioavailability. Instead, differences in manufacturing between the hydrolysates evaluated in the present study would be more likely to influence the quality of peptides generated, suggesting that the 
differences in efficacy might be mediated by differences in specific peptides generated by the hydrolytic process. Bioactive peptides have been identified in whey protein [15] and in whey protein hydrolysates [16] and have been shown to pass intact into the bloodstream in human subjects [16]. It is possible therefore that, as a yet unidentified bioactive peptide, or peptides, in $\mathrm{WPH}_{\mathrm{NB}}$ were responsible for the observed effect on the speeding of muscle damage repair.

$\mathrm{WPH}_{\mathrm{NB}}$ not only appeared to promote the most rapid recovery of muscle strength following eccentric exercise, it also elicited the highest level of fibroblast proliferation of all of the protein supplements when incubated with fibroblasts in vitro. While it is not possible to assume a causative mechanism between this in vitro effect on fibroblast proliferation and the effect on recovery of muscle strength, during tissue repair, fibroblasts are activated and proliferate to lay down new tissue matrix; thus fibroblast proliferation is an important step in the tissue repair pathway [8]. However, while $\mathrm{WPH}_{\mathrm{NB}}$ stimulated an increased proliferation of fibroblasts in vitro and accelerated tissue repair in vivo, it is not necessarily the case that the more rapid muscle repair in vivo was the result of an effect on fibroblast proliferation. Therefore, while not necessarily part of the tissue repair mechanism, the in vitro assessment of fibroblast proliferation might provide a simple quality control marker for screening different batches of $\mathrm{WPH}_{\mathrm{NB}}$ during the manufacturing process in an effort to ensure efficacy for in vivo tissue repair, even if the association is not directly causative. Additional research should evaluate the potential for fibroblast proliferation to be part of the mechanistic pathway by which $\mathrm{WPH}_{\mathrm{NB}}$ speeds recovery of muscle strength after muscle damage induced by eccentric exercise.

\section{CONCLUSIONS:}

Different methods of preparing hydrolysates from the same, or similar, starting material resulted in markedly different in vitro and in vivo effects on fibroblast proliferation and repair of skeletal muscle damage respectively. $\mathrm{WPH}_{\mathrm{NB}}$ appears superior to other hydrolysates of WPI in its ability to promote fibroblast proliferation in vitro and to speed recovery of muscle damage following eccentric exercise in vivo. $\mathrm{WPH}_{\mathrm{NB}}$ may promote post-exercise recovery and therefore facilitate the performance of greater training loads or improved exercise performance in individuals who are engaged in exercise training. Monitoring fibroblast proliferation in vitro may provide a means of ensuring quality control during the manufacture of different batches of $\mathrm{WPH}_{\mathrm{NB}}$.

Competing Interests: Andrew Brown is an employee of Murray Goulburn co-Operative Co. Ltd, the manufacturer of the supplements used in this study. The other authors have no financial interests or conflicts of interest to declare.

Authors' Contributions: All authors contributed to the study. Dale, M.J., Thomson, R.L, Coates, A.M, Howe, P.R.C., Brown, A, Buckley, J.D

Abbreviations: WPH, whey protein hyrolysate; $\mathrm{WPH}_{\mathrm{NB}}$, whey protein hydrolysate NatraBoost XR; WPI, whey protein isolate; FW, flavoured water; FBS, fetal bovine serum.

Acknowledgements and Funding: This study was supported by a grant from Murray Goulburn Co-operative Co. Ltd. 


\section{REFERENCES:}

1. Byrne C, Twist C, Eston R. Neuromuscular function after exercise-induced muscle damage. Sports Med 2004; 34: 49-69.

2. Barnett A. Using recovery modalities between training sessions in elite athletes: Does it help? . Sports Med 2006; 36: 781-796.

3. Matthews C, van Holde $\mathrm{K}$, Biochemistry. Redwood City: Benjamin Cummings Publishing Company; 1990.

4. Thomson R, Buckley J. Protein hydrolysates and tissue repair. Nutr Res Rev 2011; 24: 191-197.

5. Ziegler F, Ollivier J, Cynober L, Masini J, Coudray-Lucas C, Levy E, Goiboudeau J. Efficiency of enteral nitrogen support in surgical patients: small peptides v non-degraded proteins. Gut 1990; 31: 1277-1283.

6. Demling R, DeSanti L. Increased protein intake during the recovery phase after severe burns increases body weight gain and muscle function. J Burn Care Rehabil 1998; 19: 161-168.

7. Buckley J, Thomson R, Coates A, Howe P, DeNichilo M, Rowney M. Supplementation with a whey protein hydrolysate enhances recovery of muscle force-generating capacity following eccentric exercise. J Sci Med Sport 2010; 13: 178-181.

8. Badid C, Mounier N, Costa A, Desmoulière A. Role of myofibroblasts during normal tissue repair and excessive scarring: interest of their assessment in nephropathies. Histol Histopathol 2000; 15: 269-280.

9. Yu P, Brown A, Rowney M, Buckley J Formulation comprising whey protein and hydrolysates for improving muscle recovery. WO07/143794, 21/12/2007, 2007.

10. Altman D, Bland M. Treatment allocation by minimisation. BMJ 2005; 330: 843.

11. Naylor D. Two cheers for meta-analysis: problems and opportunities in aggregating results of clinical trials. Can Med Assoc J 1988; 138: 891-895.

12. Goldman L, Geinstein A. Anticoagulants and myocardial infarction. The problems of pooling, drowning and floating. . Ann Intern Med 1979; 90: 92-94.

13. Cooke M, Rybalka E, Stathis C, Cribb P, Hayes A. Whey protein isolate attenuates strength decline after eccentrically-induced muscle damage in healthy individuals. J Int Soc Sports Nutr 2010; 22: 30.

14. Farnfield M, Trennery C, Carey K, Cameron-Smith D. Plasma amino acid response after ingestion of different whey protein fractions. Int J Food Sci Nutr 2009; 60: 476-486.

15. Pihlanto-Leppälä A. Bioactive peptides derived from bovine whey proteins: opioid and ace-inhibitory peptides. Trends Food Sci Technol 2000; 11: 347-356.

16. Morifuji M, Koga J, Kawanaka K, Higuchi M. Branched chain amino acid-containing dipeptides, identified from whey protein hydrolysates, stimulate glucose uptake rate in L6 myotubes and isolated skeletal muscle. J Nutr Sci Vitaminol 2009; 55: 81-86. 